\title{
Chi si rivede!!! La dialisi domiciliare, questa volta quotidiana
}

\author{
Francesco P. Schena, Giovanni Pertosa
}

\section{Sezione di Nefrologia, Dipartimento dell'Emergenza e dei Trapianti di Organi, Università degli Studi di Bari, Bari}

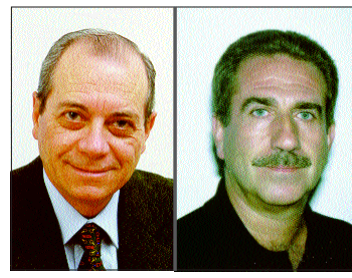

Francesco P. Schenae Giovanni Pertosa 【a dialisi è il trattamento sostitutivo che il nefrolog o utilizza per la sopravvivenza dell'uremico cronico. Parliamo di sopravvivenza; perché negli anni '60 - quando iniziò questo tipo di terapia - si cercava di sottrarre alla morte soggetti condannati per la cessazione della funzione renale. Nel corso di questi decenni la dialisi è decisamente migliorata su due aspetti: organizzativo e terapeutico. Per quanto riguarda l'aspetto organizzativo si è passati dai Centri dialisi ospedalieri a quelli ad assistenza limitata, in modo da permettere al paziente di poter avere il Centro dialisi vicino al proprio domicilio. Per l'aspetto terapeutico nuovi trattamenti dialitici e membrane sempre più biocompatibili hanno decisamente migliorato la qualità di vita del paziente. È importante sottolineare che nel corso di questi 40 anni si è cercato di trasferire il trattamento dialitico a casa del paziente per migliorare la sua permanenza in famiglia, come è avvenuto per altre malattie, in modo da utilizzare l'ospedale solo per le patologie acute.

Un notevole contributo per il trattamento domiciliare è venuto dalla dialisi peritoneale che nel nostro Paese non è stata accettata con molto entusiasmo se si considera che mediamente solo il $10 \%$ degli uremici cronici ricevono questo tipo di trattamento rispetto a percentuali molto elevate in Inghilterra e in altri Paesi europei.

Negli anni ' $70 \mathrm{fu}$ introdotta in Italia la pratica della emodialisi domiciliare (1) che in realtà non raggiunse risultati soddisfacenti, probabilmente perché i trattamenti dialitici di quell'epoca e le apparecchiature a disposizione non avevano raggiunto un elevato grado di perfezione. Ed è in virtù di questo notevole progresso tecnologico che oggi si riaffaccia all'orizzonte il trattamento emodialitico domiciliare con alcune limitazioni di cui tratteremo.

Premesso che il reinserimento del soggetto malato nella famiglia resta l'obiettivo principale, è necessario, tuttavia, che ciò avvenga non a spese dei familiari, specialmente se questi ultimi trascorrono molte ore della giornata sul luogo di lavoro. Pertanto, si deve pensare a un trattamento dialitico domiciliare che possa essere ge- stito dal paziente in piena autonomia e con profonda conoscenza di ciò che fa. Crediamo che sia giunto il momento in cui il paziente possa ricevere un trattamento dialitico quotidiano autogestito ma ugualmente controllato dal medico. Tra pochi mesi l'uremico cronico potrà disporre a casa di un proprio rene artificiale semplice e sicuro e praticare la dialisi ogni giorno per 2-3 ore. Forse è giunta l'epoca del trattamento self-service con gestione a distanza del paziente, mediante collegamento via internet con il Centro dialisi. La possibilità di utilizzare un set dialitico personalizzato e autogestito migliora, a nostro parere, le condizioni psicologiche del paziente, dà l'opportunità di una riabilitazione e una migliore qualità di vita, perché si dispone di maggiore libertà rispetto agli schemi dialitici e ai vincoli dell'ospedale. Esiste, tuttavia, un importante limite in questo nuovo tipo di trattamento: esso è rappresentato dall'accesso vascolare. Come si supererà questo limite? Alcuni gruppi di lavoro, compreso il nostro, in collaborazione con alcune società industriali stanno lavorando su questo aspetto. L'obiettivo finale sarà l'impianto di una protesi semplice che sostituisca la fistola artero-venosa e che possa essere cambiata presso il Cen- 
tro dialisi cui afferisce il paziente con un breve periodo di degenza.

A nostro parere un altro limite è rappresentato dall'accertata consapevolezza da parte del paziente che è necessario praticare il trattamento dialitico ogni giorno e non sottovalutare questo importante aspetto. Crediamo che si possa superare questo punto con un accurato addestramento alla terapia sostitutiva presso il Centro dialisi e solo dopo aver accertato la piena maturità e preparazione del paziente e dell'eventuale partner, si potrà iniziare l'emodialisi domiciliare quotidiana.

In tutto questo contesto resta da risolvere un importante problema che è quello economico. Quale sarà l'onere finanziario a carico dello Stato per un trattamento dialitico quotidiano, che viene effettuato al domicilio del paziente e prevede un controllo a distanza del paziente stesso da parte del Centro dialisi? Questo aspetto viene oggi discusso negli USA dal Medicare EJRD Program e si cerca di risolverlo con una tariffa equivalente a quattro trattamenti dialitici in ospedale. Sarà, a nostro parere, importante affrontare e discutere questo aspetto economico anche nel nostro Paese, se desideriamo diffondere e praticare il trattamento emodialitico domiciliare.

In conclusione, l'emodialisi domiciliare quotidiana, già praticata con successo da diversi gruppi di nefrologi $(2,3)$, tenderà a diffondersi sul territorio nazionale. Essa è alle porte; pertanto, è necessario organizzarsi dal punto di vista assistenziale nei Centri dialisi e sul territorio di afferenza. È importante superare lo scoglio dell'accesso vascolare, ma è anche necessario proporre al Ministero della Sanità una tariffa che tenga presente il controllo a distanza del paziente e la gestione del rene artificiale nelle sue componenti e accessori.

\section{fp.schena@nephro.uniba.it}

\section{BIBLIOGRAFIA}

1. Piccoli G, Alloatti S, Giachino G, Segoloni GP, Ragni P, Vercellone A. Primi risultati di un esperimento di dialisi domiciliare in Piemonte. Minerva Nefrologica 1972; 19: $178-84$.

2. Woods JD, Port FK, Orzol S, et al. Clinical and biochemical correlates of starting "daily" hemodialysis. Kidney Int 1999; 55: 2467-76.

3. Traeger J, Galland R, Arkouche W, Delawari E, Fouque D. Short daily hemodialysis: A four-year experience. Dial Transpl 2001; 30:7686. 